\title{
A New Optimal Homotopy Asymptotic Method for Fractional Optimal Control Problems
}

\author{
Oluwaseun Olumide Okundalaye (iD) ${ }^{1,2}$ and Wan Ainun Mior Othman (i) ${ }^{1}$ \\ ${ }^{1}$ Institute of Mathematical Sciences, University of Malaya, Kuala Lumpur 50603, Malaysia \\ ${ }^{2}$ Adekunle Ajasin University, Akungba-Akoko, Nigeria \\ Correspondence should be addressed to Oluwaseun Olumide Okundalaye; sva180049@siswa.um.edu.my
}

Received 12 November 2020; Revised 26 January 2021; Accepted 4 May 2021; Published 15 May 2021

Academic Editor: Khalil Ezzinbi

Copyright (C) 2021 Oluwaseun Olumide Okundalaye and Wan Ainun Mior Othman. This is an open access article distributed under the Creative Commons Attribution License, which permits unrestricted use, distribution, and reproduction in any medium, provided the original work is properly cited.

\begin{abstract}
Solving fractional optimal control problems (FOCPs) with an approximate analytical method has been widely studied by many authors, but to guarantee the convergence of the series solution has been a challenge. We solved this by integrating the Galerkin method of optimization technique into the whole region of the governing equations for accurate optimal values of controlconvergence parameters $C_{j}^{s}$. The arbitrary-order derivative is in the conformable fractional derivative sense. We use Euler-Lagrange equation form of necessary optimality conditions for FOCPs, and the arising fractional differential equations (FDEs) are solved by optimal homotopy asymptotic method (OHAM). The OHAM technique speedily provides the convergent approximate analytical solution as the arbitrary order derivative approaches 1 . The convergence of the method is discussed, and its effectiveness is verified by some illustrative test examples.
\end{abstract}

\section{Introduction}

The general definition of an optimal control problem necessitates minimization of an objective function of the states and control inputs of the system over a set of admissible control functions. The optimal control problems emerge naturally in different areas of science, engineering, and economics. Several significant works have been done in the area of optimal control of integer order derivative dynamic systems: the optimal control design for the hyperchaotic Chen system [1], an optimal control of time-varying and state time-delay systems using shooting method [2], a CasADi software for nonlinear optimal control problem [3], direct and simultaneous approach for constrained mixedinteger optimal control [4], a tailored algorithm for mixed integer nonlinear optimal control model of hybrid electric vehicles [5], and many more.

The arbitrary order of fractional derivative equations (FDEs) from fractional calculus has contributed a substantial part to mathematics, engineering, and different area of sciences. The previous studies review that arbitrary order derivatives provide more exact models of several engineering control systems and show the behaviour, performance, and mathematical representation of the dynamic systems in sciences than traditional calculus, for example, the positioning of satellite in space is a model using fractional order because traditional calculus is better equipped to capture the memory-like effect observed in the system. Fractional calculus applications are but not limited to liquid-containing gas bubbles [6], neurodynamics system [7], the FOCPs with a general derivative operator [8], a new adaptive synchronization and hyperchaos control of a biological snap oscillator [9], a new mathematical model for Zika virus transmission [10], the fractional features of a harmonic oscillator with position-dependent mass [11], electrohydrodynamic flow in a cylindrical conduit [12], fractional order of HIV infection model [13], and novel solution methods for IBVPs of arbitrary-order with conformable differentiation [14]. Many areas of FDEs have been researched by many authors: the multistage ADM for solving NLP problems over a nonlinear fractional dynamical system [15], the existence of the solution to FOCPs [16], 
stable analytical techniques for solving FDEs [17], Caputo-Fabrizio operator sense for brucellosis model [18], and spectral collocation method of generalized fractional operator sense for fractional calculus equations [19]. Currently, to obtain exact solutions for FOCPs is still difficult and remain a challenge. But where there is not an exact solution, appropriate approximate analytical solution (AAS) is suitable and applicable. A recent approximate analytical methods are: modified ADM for fractional optimal control problems (FOCPs) [20], optimal control of a constrained fractionally damped elastic beam [21], analyses of an optimal solutions of optimization problems from fractional gradient based system using VIM [22], conformable fractional optimal control problem of an heat conduction equations using Laplace and finite Fourier sine transforms [23], spectral Galerkin approximation [24], and direct transcription methods for FOCPs [25], but the aforementioned methods lack convergence norm that will guarantee the convergence of the series solution. In 1992, Liao proposed the homotopy analysis method (HAM) [26] for solving the nonlinear problem which was later advanced to optimal homotopy asymptotic method for noninteger order [27], new fractional homotopy method for optimal control problems (OCPs) [28], and comparisons of OHAM [29]. But OHAM has never been used to solve FOCPs, which drives this research work. We extend the work of (Alizadeh Effati, 2018a), who proposed a fractional optimal control problem with an approximate analysis solution that lacks convergence norm. We provided the solution using OHAM with an optimization technique of the Galerkin method. It is hoped that the simplicity of this formulation will initiate a new interest in the area of fractional order optimal control problems. Our focus in this paper is to widen the application of the OHAM to FOCPs. The formulation, numerical scheme, and approximate analytical results for FOCPs presented in this paper are attempts to fill this gap.

We arrange the paper as follows. In Section 2, a brief introduction to the fractional calculus and the necessary optimality conditions are discussed. In Section 3, we formulate OHAM with FOCPs and show the convergence analysis of the technique. We present numerical examples and results in Section 4. Finally, in Section 5, we present the conclusion.

\section{Fractional Calculus}

The definition of conformable fractional derivative (CFD) preserves many properties of classical order derivatives [30]. Some features that we will adopt are as follows.

Definition 1. The (left) FD starting from a function $z:[a, \infty) \longrightarrow \in$ of order $0<\alpha<1$ is defined by

$$
\left(T_{\alpha}^{a} z\right)(\psi)=\lim _{\varepsilon \longrightarrow 0} \frac{z\left(\psi+\varepsilon(\psi-a)^{1-\alpha}\right)-z(\psi)}{\varepsilon}, \quad \forall \psi>0,0<\alpha<1 .
$$

When $a=0$, we write $T_{\alpha}$. If $\left(T_{\alpha} z\right)(\psi)$ exists on $(a, b)$, then $\left(T_{\alpha}^{a} z\right)(a)=\lim _{\psi \rightarrow a^{+}}\left(T_{\alpha}^{a} z\right)(\psi)$. The (right) FD of order $0<\alpha \leq 1$ ending at $b$ of $z$ is defined by

$$
\left({ }^{b} T_{\alpha} z\right)(\psi)=-\lim _{\varepsilon \rightarrow 0} \frac{z\left(\psi+\varepsilon(b-\psi)^{1-\alpha}\right)-z(\psi)}{\varepsilon}, \quad \forall \psi>0,0<\alpha<1 .
$$

$$
\text { If }\left({ }^{b} T_{\alpha} z\right)(\psi) \quad \text { exists on }(a, b), \quad \text { then }
$$

$\left({ }^{b} T_{\alpha} z\right)(b)=\lim _{\psi \rightarrow b^{-}}\left({ }^{b} T_{\alpha} z\right)(\psi)$.

Note that if $z$ is differentiable, then $\left(T_{\alpha}^{a} z\right)(\psi)=(\psi-a)^{1-\alpha} z^{\prime}(\psi)$ and $\left({ }_{\alpha}^{b} T z\right)(\psi)=-(b-\psi)^{1-\alpha}$ $z^{\prime}(\psi)$.

Notation. $\quad\left(I_{\alpha}^{a} z\right)(\psi)=\int a^{t} z(\psi) \mathrm{d} \alpha(\psi, a)=\int a^{t}(\psi-a)^{\alpha-1}$ $z \psi \mathrm{d} \psi$. When $a=0$, we write $d \alpha(\psi)$. Similarly, in the right case, we have $\left({ }^{b} T_{\alpha} z\right)(\psi)=\int t^{b} z(\psi) \mathrm{d} \alpha(b, \psi)=$ $\int t^{b}(b-\psi)^{\alpha-1} z(\psi) \mathrm{d} \psi$. The operators $I_{\alpha}^{a}$ and ${ }^{b} I_{\alpha}$ are called conformable fractional integrals of left and right order $0<\alpha<1$.

Lemma 1. Assume that $z:[a, \infty) \longrightarrow \Re$ is continuous and $0<\alpha<1$. Then, for all $t>a$, we have

$$
T_{\alpha}^{a} I_{\alpha}^{a}=z(\psi) .
$$

In the right case, we can similarly prove the following.

Lemma 2. Assume that $z:(-\infty, b] \longrightarrow \mathfrak{R}$ is continuous and $0<\alpha<1$. Then, $\forall t<a$, we have

$$
{ }^{b} T_{\alpha}{ }^{b} I_{\alpha}=z(\psi)
$$

2.1. Necessary Optimality Condition. We define optimal control problems as a minimization of a performance index (PI) subject to system dynamic constraints on the state variable $z(t)$ and control variable $u(t)$. The necessary optimality condition helps reduce OCPs to a system of integer order ODE. The general formulation of Caputo and Riemann-Liouville derivatives is with OCPs [31]. Using the above definition equations (1) and (2), we present an Euler-Lagrange equation form for FOCPs under some consideration as follows.

Find the optimal control $u(t)$ that minimizes the performance index:

$$
J(u)=\int_{0}^{1} F(z, u, t) \mathrm{d} t,
$$

subject to the system dynamic constraints

$$
T_{\alpha}^{a} z=G(z, u, t),
$$

and the initial condition

$$
z(0)=z_{0},
$$

where $F$ and $G$ are two arbitrary functions and $T_{\alpha}^{a}$ is the conformable fractional derivative [30]. Some additional terms containing $z^{\prime} s(t)$ may be included in equation (6) at the endpoint. When $\alpha=1$, the problem above reduces to a integer OCPs [32]. Here, we take the limits of integration from 0 to 1 and consider $0.8 \leq \alpha \leq 1$. According to [33], the 
necessary equations are obtained through the combination of equations (5) and (6) and using a Lagrange multiplier approach. The variants of the resultant is taken, which is followed by using integration by parts to revise the equation so that it does not contain variants of a derivative term, enforce the necessary terminal conditions, and set the coefficients of $\delta \lambda, \delta z$, and $\delta u$ to zero. We have

$$
\begin{aligned}
T_{\alpha}^{a} z & =G(z, u, t), \\
{ }_{\alpha}^{b} T z & =\frac{\partial F}{\partial z}+\lambda \frac{\partial G}{\partial z}, \\
\frac{\partial F}{\partial u}+\lambda \frac{\partial G}{\partial u} & =0 \\
z(0) & =z_{0}, \lambda(1)=0 .
\end{aligned}
$$

Where $\lambda$ is the Lagrange multiplier. The details of the derivation of equations (8)-(11) are seen in [33]. The Euler-Lagrange equations for FOCP equations (6) and (7) represent the necessary conditions for the optimality of FOCP. The difference between arbitrary and integer OCP is that the arbitrary order FOCP consists of the left and the right FD. Examine that equation (8) contains left conformable derivative, whereas equation (9) contains the right conformable derivative. This demonstrates that the solution of OCP requires the insight of not only forward derivatives (FD) but also backward derivatives (BD) to account for end conditions. In integer-order OCP theories, this issue is either not addressed or not highlighted. This is broad because the $\mathrm{BD}$ of order 1 turns out to be negative of the FD of order 1 . It can be shown that, in the $\lim _{\alpha \longrightarrow 1}$, equations (8)-(10) goes back to those obtained using standard methods.

\section{The Formulation of OHAM with FOCPs}

We exemplify the basic concept of the OHAM by considering the following general FDEs system:

$$
\begin{aligned}
& T_{\alpha}^{a}\left(x_{k}(t)\right)+L_{k}\left(x_{k}(t)\right)+N_{k}\left(x_{k}(t)\right) \\
& \quad-g_{k}(t)=0, \quad t \in \varphi, k=1,2 \ldots m
\end{aligned}
$$

with initial conditions

$$
x_{k}(b)=a_{i},
$$

where $T_{\alpha}^{a}$ is the conformable fractional derivative, $l_{k}$ is a linear operator, $N_{k}$ is a nonlinear operator, $t$ is an independent variable, $x_{k}(t)$ is unknown function, $\varphi$ is the problem domain, and $g_{k}(t)$ is a known function. According to OHAM, one can construct a homotopy map $H_{k}\left(\phi_{i}(t, p): \varphi \times[0,1] \longrightarrow \varphi\right.$ which satisfies that equation (12) can be constructed using OHAM as [26]

$$
\begin{aligned}
& (1-\ell)\left[T_{\alpha}^{a}\left(\mathrm{~F}_{k}(t, \ell)\right)\right]=H_{k}(\ell) \\
& \quad\left[T_{\alpha}^{a} \mathrm{~F}_{k}(t, \ell)+N \mathrm{~F}_{k}(t, \ell)+L \mathrm{~F}_{k}(t, \ell)+g_{k}(t, \ell)\right],
\end{aligned}
$$

where embedding parameter $(\ell)$ is $0 \leq \ell \leq 1$, auxiliary function $H_{k}(\ell) \forall \ell \neq 0$, unknown function $\left(\mathrm{F}_{k}(t, \ell)\right)$, and
$H(0)=0$. Definitely, when $\ell=0$ and $\ell=1$, it holds that $\mathrm{F}_{k}(t, 0)=\psi_{k, 0}(t)$ and $\mathrm{F}_{k}(t, 1)=\psi_{k}(t)$, respectively. Thus, as $\ell$ moves from 0 to 1 , the solution $F_{k}(t, \ell)$ approaches from $\psi_{k, 0}(t)$ to $\psi_{k}(t)$, where initial guess $\psi_{k, 0}(t)$ satisfies the linear operator generated from equation (14) for $\ell=0$ as

$$
T_{\alpha}^{a}\left(\psi_{k, 0}(t)\right)=0, \quad \psi_{k, 0}(b)=0
$$

$H_{k}(\ell)$ is given as

$$
H_{k}(\ell)=\sum_{j=1}^{n} \ell^{j} C_{j}
$$

where $C_{j}^{s}$ can be known later. We get approximate solution by expanding $\mathrm{F}_{k}\left(t, \ell, C_{j}\right)$ in Taylor's series in terms of $\ell$

$$
\mathrm{F}_{k}\left(t, \ell, C_{j}\right)=\psi_{k, 0}(t)+\sum_{k \geq 1} \psi_{i, k}\left(t, C_{j}\right) \ell^{i}, \quad j=1,2, \ldots, n,
$$

using equation (14) with collections of the coefficient like power of $\ell$, gives the governing equations $\psi_{i, 0}(t)$ in a linear form in equation (15). Then, the $1^{\text {st }}$ problem is given as

$$
T_{\alpha}^{a}\left(\psi_{k, 1}(t)\right)+g_{k}(t)=C_{1} N_{0}\left(\psi_{k, 0}(t)\right), \psi_{k, 1}(b)=0 .
$$

The general governing equations for $\psi_{k, i}(t)$ is

$$
\begin{gathered}
T_{\alpha}^{a}\left(\psi_{k, i}(t)\right)-T_{\alpha}^{a}\left(\psi_{k, i-1}(t)\right)=C_{i} N_{k, 0}\left(\psi_{k, 0}(t)\right)+\sum_{m=1}^{i-1} C_{j, m} \\
{\left[T_{\alpha}^{a}\left(\psi_{k, i-m}(t)\right)+N_{k, i-m}\left(\psi_{k, i-1}(t)\right)\right]} \\
\psi_{k, i}(b)=0, \quad i=2,3, \ldots, m
\end{gathered}
$$

where $N_{k, m}\left(\psi_{0}(t), \psi_{k, 1}(t), \ldots, \psi_{k, m}(t)\right)$ is the coefficient of $\ell^{m}$, produced by expanding $N_{k}\left(\mathrm{~F}_{k}\left(t, \ell, C_{j}\right)\right)$ in series relating to $\ell$

$$
N_{k}\left(F_{k}\left(t, \ell, C_{j}\right)\right)=N_{k, 0}\left(\psi_{k, 0}(t)\right)+\sum_{m \geq 1} N_{k, m}\left(\psi_{0}, \psi_{1}, \ldots, \psi_{m}\right) \ell^{m} .
$$

The convergence of series solution equation (21) relies on $C_{j}^{s}$. If it is convergent at $\ell=1$, it gives solution to equation (12) as

$$
\psi_{k}\left(t, C_{j}\right)=\psi_{k, 0}(t)+\sum_{k \geq 1}^{m} \psi_{i, k}\left(t, C_{j}\right), \quad j=1,2, \ldots, n .
$$

Using equation (22) in equation (12), we have expression for the residual error as

$$
\begin{aligned}
R_{k}\left(t, C_{j}\right)= & T^{\alpha}\left(\psi_{k}\left(t, C_{j}\right)\right)+L_{k}\left(\psi_{k}\left(t, C_{j}\right)\right) \\
& +N_{k}\left(\psi_{k}\left(t, C_{j}\right)-g_{k}(t)\right) .
\end{aligned}
$$

If

$$
R_{k}\left(t, C_{j}\right)=0,
$$


then $\widetilde{\psi}_{k}\left(t, C_{j}\right)$ is the exact solution. Usually, such a case does not occur. We adopt Galerkin method to find the optimal values $C_{j}^{s}$ as follows:

$$
\ell_{k}=\frac{\partial \widetilde{\psi}_{k}\left(t, C_{j}\right)}{\partial C_{j}}=0, \quad k=1,2, \ldots, m
$$

Minimize the functional

$$
\Delta_{k}\left(C_{j}\right)=\int_{a}^{b} \ell_{k} \times R_{k}\left(t, C_{j}\right) \mathrm{d} t,
$$

where the values of $a$ and $b$ depend on the given problem. With these known $C_{j}^{s}$, the approximate analytical solution equation (12) is well known.

We calculate the correctness of OHAM by

(1) Error norm $L_{2}$

$$
L_{2}=\left\|\Psi^{\text {exact }}-\Psi_{N}\right\| \approx \sqrt{\frac{b-a}{N} \sum_{k=0}^{N}\left|\psi_{k}^{\text {exact }}-\left(\psi_{N}\right)_{k}\right|^{2}}
$$

(2) Error norm $L_{\infty}$

$$
L_{\infty}=\left\|\Psi^{\text {exact }}-\Psi_{N}\right\|_{\infty} \approx \max _{k}\left|\psi_{k}^{\text {exact }}-\left(\psi_{N}\right)_{k}\right| .
$$

\subsection{Convergence Analysis of the Method}

Theorem 3.1. If the series $\psi_{k}\left(t, C_{j}\right)=\psi_{k, 0}(t)+\sum_{i=1}^{m}$ $\psi_{k, i}\left(t, C_{j}\right)$, for $j=1,2, \ldots, n$, converges where $\psi_{k}\left(t, C_{j}\right)$ is governed by equation (22) under the definitions equations (19) and (20), it achieves the solution of equations (12) and (13).

Proof. If we assume $\sum_{m=1}^{\infty} \psi_{k, m}\left(t, C_{j}\right)$, for $k=1,2, \ldots, n$, converges to $\psi_{k}\left(t, C_{j}\right)$, then

$$
\lim _{m \longrightarrow \infty} \psi_{k, m}\left(t, C_{j}\right)=0, \quad \forall k=1,2, \ldots, n .
$$

From equation (19), we can write

$$
\begin{aligned}
& \sum_{i=1}^{\infty}\left[C_{i} N_{k, 0}\left(x_{k, 0}(t)\right)+\sum_{m=1}^{i-1} C_{j, m}\left[T_{\alpha}^{a}\left(\psi_{k, i-m}(t)\right)+N_{k, i-m}\left(\psi_{k, i-1}(t)\right)\right]\right. \\
& =\sum_{k=1}^{\infty}\left[T_{\alpha}^{a}\left(\psi_{i, k}(t)\right)-T_{\alpha}^{a}\left(\psi_{i, k-1}(t)\right)(t)\right], \\
& =\lim _{n \longrightarrow \infty} \sum_{k=1}^{n} T_{\alpha}^{a}\left(\psi_{i, k}(t)\right)-T_{\alpha}^{a}\left(\psi_{i, k-1}(t)\right), \\
& =T_{\alpha}^{a} \psi_{11}(t)+\left(T_{\alpha}^{a} \psi_{22}(t)-T_{\alpha}^{a} \psi_{21}(t)\right)+\cdots+\left(T_{\alpha}^{a} \psi_{n n}(t)-T_{\alpha}^{a} x_{n(n-1)}(t)\right), \\
& =T_{\alpha}^{a}\left[\lim _{n \longrightarrow \infty} \sum_{m=1}^{n} \psi_{n n}(t)\right]=T_{\alpha}^{a}\left[\lim _{n \longrightarrow \infty} \psi_{n n}(t)\right]=0 .
\end{aligned}
$$

Equate the RHS of equation (33) with the following equation:

$$
\begin{aligned}
& \left.\left.0=\sum_{m=1}^{\infty}\left[T_{\alpha}^{a} \psi_{k(m-1)}+N \psi_{k(m-1)} L \psi_{k(m-1)}+N \psi_{k(m-1)}-g_{k}(t)\right)\right)\right], \\
& \left.\sum_{m=1}^{\infty}\left[T_{\alpha}^{a} \psi_{k(m-1)}+L \psi_{k(m-1)}+N \psi_{k(m-1)}\right)\right]=g_{k}(t), \\
& T_{\alpha}^{a} \psi_{k}\left(t, C_{j}\right)+L \psi_{k}\left(t, C_{j}\right)+N \psi_{k}\left(t, C_{j}\right)-g_{k}(t)=0, \quad \forall k=1,2, \ldots, n .
\end{aligned}
$$

If the $C_{j}$ is chosen properly, then equation (36) leads to the solution of equations (12) and (13) 


\section{Numerical Example and Results}

Example 1. Find the control $u(t)$ that minimizes the quadratic performance index

$$
J(u)=\frac{1}{2} \int_{0}^{1}\left[z^{2}(t)+u^{2}\right] \mathrm{d} t,
$$

subject to system dynamics

$$
T_{\alpha}^{a}=-z+u
$$

and the initial condition

$$
z(0)=1 \text {. }
$$

The exact solution for the system for $\alpha=1$ is given as

$$
\begin{aligned}
& z(t)=\cosh (\sqrt{2 t})+\beta \sinh (\sqrt{2 t}), \\
& u(t)=(1+\sqrt{2 \beta}) \cosh (\sqrt{2 t})+(\sqrt{2}+\beta) \sinh (\sqrt{2 t}),
\end{aligned}
$$

where

$$
\beta=\frac{\cosh (\sqrt{2}+\sqrt{2 \sinh }(\sqrt{2})}{\sqrt{2 \cosh (\sqrt{2}+\sinh (\sqrt{2})}} \approx-0.98 .
$$

Following equations (8)-(11) procedure, we have

$$
\begin{gathered}
T_{\alpha}^{a} z=-z+u, \\
{ }^{b} T_{\alpha}^{a} u=-z-u, \\
z(0)=1, u(1)=0 .
\end{gathered}
$$

Using OHAM, the solutions for fractional-order are acquired. We choose the linear and nonlinear operators in the following forms:

$$
\begin{aligned}
L_{1}\left[\mathrm{~F}_{1}(t, \ell)\right] & =T_{\alpha}^{a} \mathrm{~F}_{1}(t, \ell), \\
L_{2}\left[\mathrm{~F}_{2}(t, \ell)\right] & ={ }^{b} T_{\alpha} \mathrm{F}_{2}(t, \ell), \\
N_{1}\left[\mathrm{~F}_{1}(t, \ell)\right] & =T_{\alpha}^{a} \mathrm{~F}_{1}(t, \ell)+\mathrm{F}_{1}(t, \ell)+\mathrm{F}_{2}(t, \ell), \\
N_{2}\left[\mathrm{~F}_{2}(t, \ell)\right] & ={ }^{b} T_{\alpha} \mathrm{F}_{2}(t, \ell)-\mathrm{F}_{1}(t, \ell)-\mathrm{F}_{2}(t, \ell) .
\end{aligned}
$$

Using homotopy in equation (14),

$$
(1-\ell) T_{\alpha}^{a} \mathrm{~F}_{1}(t, \ell)=H_{k}(\ell)\left[T_{\alpha}^{a} \mathrm{~F}_{1}(t, \ell)+\mathrm{F}_{1}(t, \ell)+\mathrm{F}_{2}(t, \ell)\right],
$$

$$
(1-\ell)^{b} T_{\alpha} \mathrm{F}_{2}(t, \ell)=H_{k}(\ell)\left[{ }^{b} T_{\alpha} \mathrm{F}_{2}(t, \ell)-\mathrm{F}_{1}(t, \ell)-\mathrm{F}_{2}(t, \ell],\right.
$$

where

$$
\begin{aligned}
& F_{1}(t, \ell)=z_{0}(t)+\sum_{j \leq 1} z_{1, j}(t) \ell^{j}, \\
& F_{2}(t, \ell)=u_{0}(t)+\sum_{j \leq 1} u_{1, j}(t) \ell^{j},
\end{aligned}
$$

$$
\begin{aligned}
& H_{k}(\ell)=\ell C_{1}+\ell^{2} C_{2}+\ell^{3} C_{3} \\
& \quad+\cdots \ell^{m} C_{m}, \quad k=1,2, \ldots, m .
\end{aligned}
$$

Substituting $\mathrm{F}_{1}(t, \ell), \mathrm{F}_{2}(t, \ell)$, and $H_{k}(\ell)$ into equations (47) and (48), and equating the coefficient of likes power of $\ell$, it gives linear FDEs as follows:

$$
\begin{aligned}
& \ell^{0}: T_{\alpha}^{a} z_{0}(t)=0 \\
& \ell^{0}:{ }^{b} T_{\alpha} u_{0}(t)=0 \\
& \ell^{1}: T_{\alpha}^{a} z_{1}(t)=T_{\alpha}^{a} z_{0}(t) C_{1}-T_{\alpha}^{a} z_{0}(t) \\
& \quad+z_{0}(t) C_{1}+u_{0}(t) C_{1}=0 \\
& \ell^{1}:{ }^{b} T_{\alpha} u_{1}(t)={ }^{b} T_{\alpha} u_{0}(t) C_{1}-T_{\alpha}^{a} u_{0}(t) \\
& \quad+z_{0}(t) C_{1}+u_{0}(t) C_{1}=0 \\
& \ell^{2}: T_{\alpha}^{a} z_{2}(t)=T_{\alpha}^{a} z_{0}(t) C_{2}+T_{\alpha}^{a} z_{1}(t) C_{1} \\
& \quad-T_{\alpha}^{a} z_{1}(t)+z_{0}(t) C_{2}+z_{1}(t) C_{1} \\
& \quad+u_{0}(t) C_{2}+u_{1}(t) C_{1}=0 \\
& \ell^{2}:{ }^{b} T_{\alpha} u_{2}(t)={ }^{b} T_{\alpha} u_{0}(t) C_{2}+{ }^{b} T_{\alpha} u_{1}(t) C_{1} \\
& \quad-{ }^{b} T_{\alpha} u_{1}(t)-u_{0}(t) C_{2}-u_{1}(t) C_{1} \\
& +z_{0}(t) C_{2}+z_{1}(t) C_{1}=0 .
\end{aligned}
$$

Using the operator Lemmas 1 and 2 on the above equations with initial condition gives

$$
\begin{gathered}
z_{0}(t)=0 \\
u_{0}(t)=0 \\
z_{1}\left(t, C_{1}\right)=-5 t^{1 / 5} C_{1}+1, \\
u_{1}\left(t, C_{1}\right)=5 t^{1 / 5} C_{1}, \\
z_{2}\left(t, C_{1}, C_{2}\right)=5 t^{1 / 5} C_{1}^{2}-2 C_{1}+C_{2}+1, \\
u_{2}\left(t, C_{1}, C_{2}\right)=-25 t^{2 / 5} C_{1}^{2}-5 t^{1 / 5} C_{1}^{2} \\
+10 t^{1 / 5} C_{1}+5 C_{2} t^{1 / 5} \ldots
\end{gathered}
$$

Summing up the solution from equations (58)-(63), the $3^{\text {rd }}$-order approximate analytical method generated by OHAM, for $\ell=1$, is

$$
z\left(t, C_{1}, C_{2}\right)=\left(5 C_{1}^{2}-15 C_{1}-5 C_{2}\right) t^{1 / 5}+3
$$

$$
u\left(t, C_{1}, C_{2}\right)=\left(15 C_{1}-5 C_{1}^{2}+5 C_{2}\right) t^{1 / 5}-25^{2 / 5} C_{1}^{2}
$$

We determine $C_{1}$ and $C_{2}$ by using the procedure mentioned in equations (24)-(26).

For $z(t)$, 


$$
\begin{aligned}
& C_{1}=-0.2553704121, \\
& C_{2}=1.408561895 .
\end{aligned}
$$

And, for $u(t)$,

$$
\begin{aligned}
& C_{1}=-0.1925118854, \\
& C_{2}=1.0134328546 .
\end{aligned}
$$

Using the $3^{\text {rd }}$-order approximate analytical solution given in equations (64) and (65), we have

$$
\begin{aligned}
& z(t)=-4.591553468 t^{1 / 5}+3 \\
& u(t)=-0.9265206505 t^{2 / 5}+1.994181864 t^{1 / 5} .
\end{aligned}
$$

Figure 1 shows the approximate analytical solutions of the state variable $z(t)$ and control variable $u(t)$ at different values of $\alpha$ using OHAM after the $3^{\text {rd }}$ order of approximate solution. Table 1 shows the control-convergence parameter at different values of fractional-order for both state and control variables, Table 2 shows comparisons and absolute errors between the proposed method and the exact solution at integer order $\alpha=1$, and Table 3 shows the optimal values of the cost function and the comparisons between OHAM and VIM. It is observed that OHAM converges to optimal values faster in CPU times and iterations.

Example 2. Considering the following problem of minimising the functional

$$
J(u)=\frac{1}{2} \int_{0}^{1}\left[z_{1}(t)^{2}+z_{2}(t)^{2}+u(t)^{2}\right] \mathrm{d} t,
$$

subjected to the dynamic constraints

$$
\begin{aligned}
& T_{\alpha}^{a} z_{1}(t)=-z_{1}(t)+z_{2}(t)+u(t), \\
& { }^{b} T_{\alpha} z_{2}(t)=-2 z_{2}(t),
\end{aligned}
$$

and the initial conditions

$$
z_{1}(0)=z_{2}(0)=1 \text {. }
$$

The exact solution for this system for $\alpha=1$ is

$$
\begin{aligned}
& z_{1}(t)=\frac{-3}{2} e^{-2 t}+2.48164 e^{-\sqrt{2 t}}+0.018352 e^{\sqrt{2 t}}, \\
& z_{2}(t)=e^{-2 t}, \\
& u(t)=\frac{1}{2} e^{-2 t}-1.027934 e^{-\sqrt{2 t}}+0.0443056 e^{\sqrt{2 t}} .
\end{aligned}
$$

For this example, the necessary optimality conditions are as follows:

$$
\begin{aligned}
T_{\alpha}^{a} z_{1}(t)-z_{1}(t)+z_{2}(t)+u(t) & =0, \\
{ }^{b} T_{\alpha} z_{2}(t)+2 z_{2}(t) & =0, \\
u(t)+p_{1}(t) & =0, \\
T_{\alpha}^{a} p_{1}(t)+p_{(t)}-z_{1}(t) & =0, \\
{ }^{b} T_{\alpha} p_{2}(t)-2 p_{2}(t)+p_{1}(t)+z_{2}(t) & =0, \\
z_{1}(0) & =z_{2}(0)=1, \\
p_{1}(1) & =p_{2}(1)=0 .
\end{aligned}
$$

Following the same procedure above, we have

$$
z_{1}\left(t, C_{1}, C_{2}\right)\left(-30 C_{1}+10 C_{1}^{2}-10 C_{2}\right) t^{1 / 10}+50 t^{1 / 5} C_{1}^{2}+3
$$

$$
\begin{aligned}
& z_{2}\left(t, C_{1}, C_{2}\right)=\left(30 C_{1}+90 C_{1}^{2}+10 C_{2}\right) t^{1 / 10}-30 C_{1}-50 \\
& \quad \times t^{1 / 5} C_{1}^{2}-40 C_{1}^{2}-10 C_{2},
\end{aligned}
$$

$$
\begin{aligned}
& u\left(t, C_{1}, C_{2}\right)=\left(30 C_{1}+110 C_{1}^{2}+10 C_{2}\right) t^{1 / 10}-30 C_{1}-100 \\
& \quad \times t^{1 / 5} C_{1}^{2}-10 C_{1}^{2}-10 C_{2} .
\end{aligned}
$$

We determine $C_{1}$ and $C_{2}$ by using the procedure mentioned in equations (24)-(26). For $z_{1}(t)$,

$$
\begin{aligned}
& C_{1}=1.143999690 \times 10^{-9}, \\
& C_{2}=0.2950819638,
\end{aligned}
$$

For $z_{2}(t)$,

$$
\begin{aligned}
& C_{1}=-0.1820636120, \\
& C_{2}=0.2099839413,
\end{aligned}
$$

and $u(t)$

$$
\begin{aligned}
& C_{1}=-6.577999908 \times 10^{-10}, \\
& C_{2}=0.3913043497
\end{aligned}
$$

Using the $3^{\text {rd }}$-order approximate analytical solution given in equations (83)-(85), we have

$$
\begin{aligned}
& z_{1}(t)=-2.950819672 \times t^{1 / 10}+3.01234+6.543676455 \times 10^{-17} \times t^{1 / 5}, \\
& z_{2}(t)=-0.378824654 \times t^{1 / 10}+2.036182595-1.657357940 \times t^{1 / 5},
\end{aligned}
$$




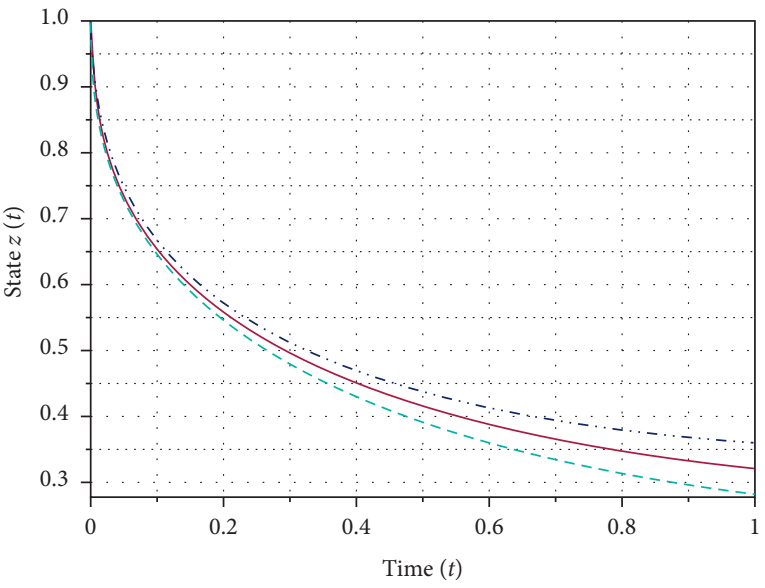

(a)

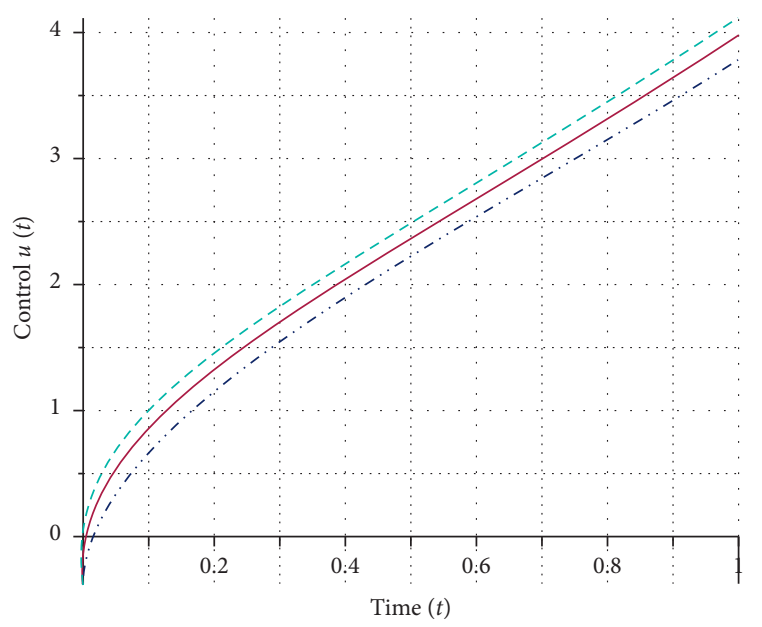

(b)

Figure 1: (a, b) For different values of $\alpha$ (exact $=$ dot, $\alpha=1$ : long dash, $\alpha=0.9$ : solid, and $\alpha=0.8$ : dash).

TABle 1: Control-convergence parameters $C_{j}^{s}$ at different values of $\alpha$.

\begin{tabular}{lcccc}
\hline Variable & $z(t)$ & $z(t)$ & $u(t)$ & $u(t)$ \\
\hline$\alpha$ & $C_{1}$ & $C_{2}$ & $C_{1}$ & $C_{2}$ \\
1 & -0.2553704121 & 1.408561895 & -0.1925118854 & 1.0134328546 \\
0.9 & -0.282311385 & 1.354541654 & -0.2136217553 & 0.9134328546 \\
0.8 & -0.312012513 & 1.319562884 & -0.2414217624 & 0.8134328546 \\
\hline
\end{tabular}

TABle 2: Comparisons and absolute error between (OHAM and exact, $\alpha=1$ ).

\begin{tabular}{lcccccc}
\hline$t_{\mathrm{k}}$ & OHAM $z(t)$ & OHAM $u(t)$ & Exact $z(t)$ & Exact $u(t)$ & Error $z(t)$ & Error $u(t)$ \\
\hline 0.0 & 1.00000000 & 0.00000000 & 1.00000000 & 0.00000000 & 0.00000000 & 0.00000000 \\
0.1 & 0.667606464 & 0.68114171 & 0.667144195 & 0.681603987 & 0.000462277 & 0.000462269 \\
0.2 & 0.572490044 & 1.15082042 & 0.571814575 & 1.151495891 & 0.000675469 & 0.000675471 \\
0.3 & 0.513009607 & 1.53717622 & 0.512155196 & 1.538030634 & 0.000854411 & 0.000854414 \\
0.4 & 0.470973769 & 1.88672253 & 0.469955226 & 1.887741076 & 0.001018543 & 0.001018546 \\
0.5 & 0.451473134 & 2.21698046 & 0.443945101 & 2.218155658 & 0.007528033 & 0.001175198 \\
0.6 & 0.439567403 & 2.53673706 & 0.438392205 & 2.538065126 & 0.001175198 & 0.001328066 \\
0.7 & 0.415403119 & 2.85112316 & 0.414075062 & 2.852602442 & 0.001328057 & 0.001479282 \\
0.8 & 0.396528946 & 3.16344266 & 0.395049669 & 3.165072917 & 0.001479277 & 0.001630257 \\
0.9 & 0.38171058 & 3.47597703 & 0.38008033 & 3.477758981 & 0.00163025 & 0.001781951 \\
1 & 0.36841575 & 3.79138812 & 0.368333813 & 3.792323188 & $8.1937 \mathrm{E}-05$ & 0.000935068 \\
\hline
\end{tabular}

TABLE 3: Optimal values of $J$ at different choices of $\alpha$ for example 1.

\begin{tabular}{lcc}
\hline$\alpha$ & VIM Ali et al. (2018) & OHAM \\
\hline 1.0 & 0.1929093 & 0.1929090 \\
0.9 & 0.17953 & 0.17152 \\
0.8 & 0.16711 & 0.16709 \\
\hline
\end{tabular}

$$
u(t)=3.913043477 \times t^{1 / 10}-3.913043477-4.327008279 \times 10^{-17} \times t^{1 / 5} .
$$

Figure 2 shows the approximate analytical solutions of the states variables $z_{1}(t)$ and $z_{2}(t)$ and control variable $u(t)$ at different values of $\alpha$ using OHAM after the $3^{\text {rd }}$ order of approximate solution. Tables 4 and 5 show the controlconvergence parameter at different values of fractional-order for both states and control variables, Table 6 shows 


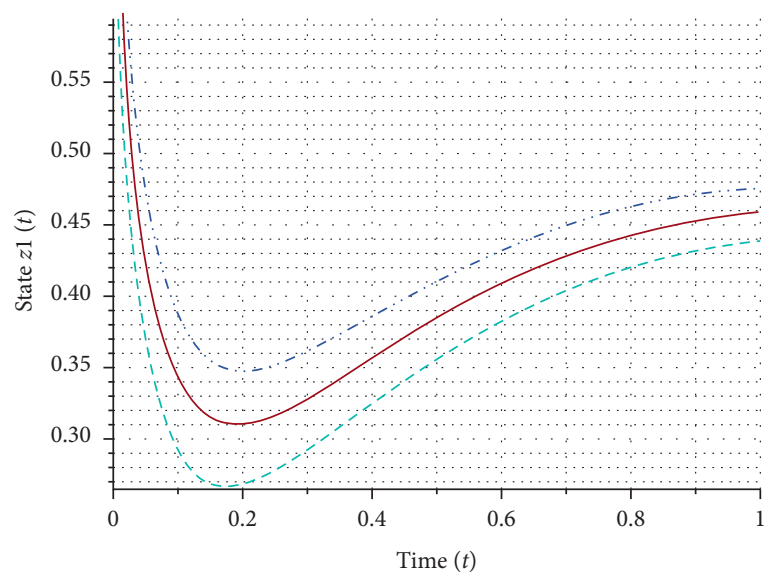

(a)

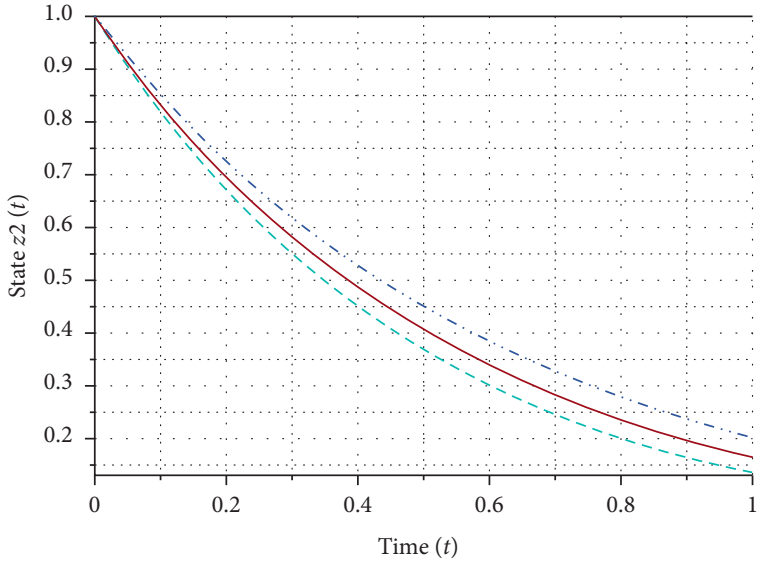

(b)

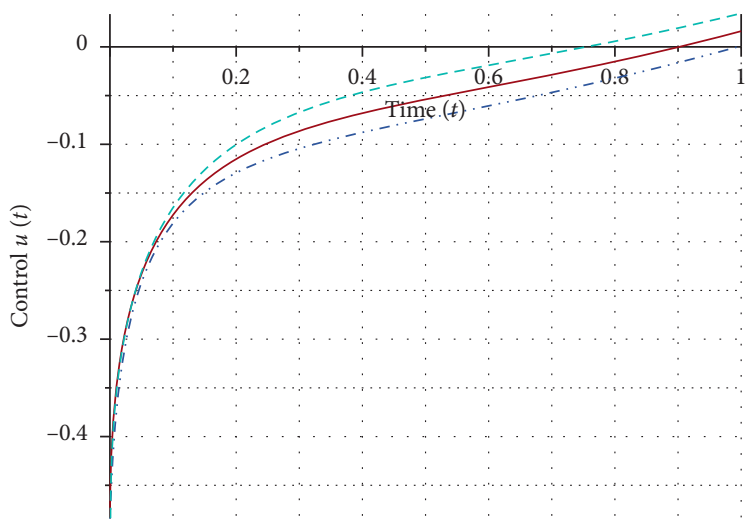

(c)

Figure 2: $(\mathrm{a}-\mathrm{c})$ For different values of $\alpha$ (exact $=$ dot, $\alpha=1$ : long dash, $\alpha=0.9$ : solid, and $\alpha=0.8$ : dash).

TABLE 4: Control-convergence parameters $C_{j}^{s}$ at different values of $\alpha$.

\begin{tabular}{lcccc}
\hline Variable & $z_{1}(t)$ & $z_{1}(t)$ & $z_{2}(t)$ & $z_{2}(t)$ \\
\hline$\alpha$ & $C_{1}$ & $C_{2}$ & $C_{1}$ & $C_{2}$ \\
1 & $1.143999690 \times 10^{-9}$ & 0.2950819638 & -0.1820636120 & 0.2099839413 \\
0.9 & $1.113328281 \times 10^{-9}$ & 0.2536726322 & -0.2121547231 & 0.1845623225 \\
0.8 & $1.091424280 \times 10^{-9}$ & 0.1963215213 & -0.2431545343 & 0.159436311 \\
\hline
\end{tabular}

TABLE 5: Control-convergence parameters $C_{j}^{s}$ at different values of $\alpha$.

\begin{tabular}{lcr}
\hline Variable & $u(t)$ & $u(t)$ \\
\hline$\alpha$ & $C_{1}$ & $C_{2}$ \\
1 & $-6.577999908 \times 10^{-10}$ & 0.3913043497 \\
0.9 & $-6.661323216 \times 10^{-10}$ & 0.3612134421 \\
0.8 & $-6.755673906 \times 10^{-10}$ & 0.3125152370 \\
\hline
\end{tabular}

TABle 6: Comparisons of (OHAM and exact, $\alpha=1$ ).

\begin{tabular}{lcccccc}
\hline$t_{k}$ & OHAM $z_{1}(t)$ & OHAM $z_{2}(t)$ & OHAM $u(t)$ & Exact $z_{1}(t)$ & Exact $z_{2}(t)$ & Exact $u(t)$ \\
\hline 0.0 & 1.0000000 & 0.00000000 & 1.0000000 & 0.00000000 & 0.0000000 & 0.00000000 \\
0.1 & 0.388025392 & 0.852996359 & -0.178200169 & 0.387385984 & 0.852143789 & -0.178609739 \\
0.2 & 0.348055508 & 0.727602788 & -0.126901219 & 0.347524221 & 0.726149037 & -0.127572209 \\
0.3 & 0.360826387 & 0.620642529 & -0.102402516 & 0.360365496 & 0.618783392 & -0.10322697 \\
0.4 & 0.385903334 & 0.529405818 & $-8.63 E-02$ & 0.385494492 & 0.527292424 & $-8.72 E-02$ \\
0.5 & 0.385903334 & 0.451581235 & $-7.29 E-02$ & 0.385494492 & 0.449328964 & $-7.38 E-02$ \\
0.6 & 0.411381137 & 0.385197149 & $-5.97 E-02$ & 0.411013257 & 0.382892886 & $-6.06 E-02$ \\
0.7 & 0.433264531 & 0.328571766 & $-4.68 E-02$ & 0.432930139 & 0.326279795 & $-4.69 E-02$ \\
0.8 & 0.450436393 & 0.28027052 & $-3.21 E-02$ & 0.4501301 & 0.278037301 & $-3.22 E-02$ \\
0.9 & 0.46293519 & 0.239069733 & $-1.65 E-02$ & 0.462652925 & 0.236927759 & $-1.66 E-02$ \\
1 & 0.47099984 & 0.207193943 & $-1.69 E-03$ & 0.470997167 & 0.205152843 & $-1.70 E-03$ \\
\hline
\end{tabular}


TABLE 7: Absolute error between (OHAM and exact, $\alpha=1$ ).

\begin{tabular}{lcc}
\hline Error $z_{1}(t)$ & Error $z_{2}(t)$ & Error $u(t)$ \\
\hline 0.000000 & 0.000000 & 0.000000 \\
0.000639408 & 0.00085257 & 0.00040957 \\
0.000531287 & 0.001453751 & 0.00067099 \\
0.000460891 & 0.001859137 & 0.000824454 \\
0.000408842 & 0.002113394 & 0.0009 \\
0.000408842 & 0.002252271 & 0.0009 \\
0.00036788 & 0.002304263 & 0.0009 \\
0.000334392 & 0.002291971 & $1 E-04$ \\
0.000306293 & 0.002233219 & 0.0001 \\
0.000282265 & 0.002141974 & $1 E-04$ \\
$2.673 E-06$ & 0.0020411 & $1 E-05$ \\
\hline
\end{tabular}

TABLe 8: Optimal values of $J$ at different choices of $\alpha$.

\begin{tabular}{lcc}
\hline$\alpha$ & VIM Ali et al, (2018) & OHAM \\
\hline 1.0 & 0.4319089 & 0.4319088 \\
0.9 & 0.4030897 & 0.4030893 \\
0.8 & 0.3760124 & 0.3760122 \\
\hline
\end{tabular}

comparisons between OHAM and the exact solution at integer order $\alpha=1$, Table 7 shows absolute errors between the proposed method and the exact solution at integer order at $\alpha=1$, and Table 8 shows the optimal values of the cost function and the comparisons between OHAM and VIM. It is observed that OHAM converges to optimal values faster in CPU times and iterations.

\section{Conclusion}

In this research article, we examine the effectiveness of the OHAM to generate an accurate approximate analytical solution to FOCPs. We derive the necessary optimal condition and then solve the system of nonlinear FDEs with OHAM. The technique helps provide an accurate approximate analytical solution to the problems. The fractional derivative operator is in the conformable fractional derivative operator sense. We show that Galerkin method helps to determine the optimal values of the control-convergence parameter. It can be seen that the OHAM results corresponded with the exact solution at $\alpha=1$, which shows the effectiveness of the proposed method to provide an accurate approximate analytical solution. OHAM is used to solve this type of problems for the first time and gave optimal values to the convergence norm challenge for accurate series solution. No existing approximate solution is known to have considered such a nonlinear constrained programming problem with OHAM ahead of this one. OHAM technique is very easy to implement and efficient for solving FOCPs.

\section{Data Availability}

No data were used to support this study.

\section{Conflicts of Interest}

The authors declare that they have no conflicts of interest.

\section{Authors' Contributions}

All authors have equal contributions and they read and approved the final version of the paper.

\section{References}

[1] S. Effati, H. S. Nik, and A. Jajarmi, "Hyperchaos control of the hyperchaotic chen system by optimal control design," Nonlinear Dynamics, vol. 73, no. 1-2, pp. 499-508, 2013.

[2] A. Jajarmi and M. Hajipour, "An efficient recursive shooting method for the optimal control of time-varying systems with state time-delay," Applied Mathematical Modelling, vol. 40, no. 4, pp. 2756-2769, 2016.

[3] J. A. E. Andersson, J. Gillis, G. Horn, J. B. Rawlings, and M. Diehl, "CasADi: a software framework for nonlinear optimization and optimal control," Mathematical Programming Computation, vol. 11, no. 1, pp. 1-36, 2019.

[4] C. Kirches, F. Lenders, and P. Manns, "Approximation properties and tight bounds for constrained mixed-integer optimal control," SIAM Journal on Control and Optimization, vol. 58, no. 3, pp. 1371-1402, 2020.

[5] N. Robuschi, C. Zeile, S. Sager, and F. Braghin, "Multiphase mixed-integer nonlinear optimal control of hybrid electric vehicles,” Automatica, vol. 123, Article ID 109325, 2021.

[6] A. K. Gupta and S. Saha Ray, "On the solitary wave solution of fractional Kudryashov-Sinelshchikov equation describing nonlinear wave processes in a liquid containing gas bubbles," Applied Mathematics and Computation, vol. 298, pp. 1-12, 2017.

[7] Y. Zheng and Z. Zhao, "The discontinuous Galerkin finite element method for fractional cable equation," Applied $\mathrm{Nu}$ merical Mathematics, vol. 115, pp. 32-41, 2017.

[8] A. Jajarmi and D. Baleanu, "On the fractional optimal control problems with a general derivative operator," Asian Journal of Control, vol. 23, no. 2, pp. 1062-1071, 2019.

[9] S. S. Sajjadi, D. Baleanu, A. Jajarmi, and H. M. Pirouz, "A new adaptive synchronization and hyperchaos control of a biological snap oscillator," Chaos, Solitons \& Fractals, vol. 138, Article ID 109919, 2020.

[10] R. Shahram, M. Hakimeh, and J. Amin, "A new mathematical model for Zika virus transmission," Advances in Difference Equations, vol. 2020, p. 589, 2020.

[11] D. Baleanu, A. Jajarmi, S. S. Sajjadi, and J. H. Asad, "The fractional features of a harmonic oscillator with positiondependent mass," Communications in Theoretical Physics, vol. 72, no. 5, p. 055002, 2020.

[12] S. Thirumalai and R. Seshadri, "Spectral solutions of fractional differential equation modelling electrohydrodynamics flow in a cylindrical conduit," Communications in Nonlinear Science and Numerical Simulation, vol. 79, Article ID 104931, 2019.

[13] A. Boukhouima, K. Hattaf, and N. Yousfi, "Dynamics of a fractional order HIV infection model with specific functional response and cure rate," International Journal of Differential Equations, vol. 2017, Article ID 8372140, 8 pages, 2017.

[14] M. Yavuz, "Novel solution methods for initial boundary value problems of fractional order with conformable 
differentiation," An International Journal of Optimization and Control: Theories \& Applications (IJOCTA), vol. 8, no. 1, pp. 1-7, 2017.

[15] F. Evirgen and N. Özdemir, "Multistage adomian decomposition method for solving NLP problems over a nonlinear fractional dynamical system," Journal of Computational and Nonlinear Dynamics, vol. 6, no. 2, pp. 1-6, 2011.

[16] R. Kamocki, "On the existence of optimal solutions to fractional optimal control problems," Applied Mathematics and Computation, vol. 235, pp. 94-104, 2014.

[17] X.-J. Yang, M. Abdel-Aty, and C. Cattani, "A new general fractional-order derivataive with Rabotnov fractional-exponential kernel applied to model the anomalous heat transfer," Thermal Science, vol. 23, no. 3, pp. 1677-1681, 2019.

[18] O. J. Peter, "Transmission dynamics of fractional order Brucellosis model using caputo-fabrizio operator," International Journal of Differential Equations, vol. 2020, Article ID 2791380, 11 pages, 2020.

[19] Q. Xu and Z. Zheng, "Spectral collocation method for fractional differential/integral equations with generalized fractional operator," International Journal of Differential Equations, vol. 2019, Article ID 3734617, 14 pages, 2019.

[20] A. Alizadeh and S. Effati, "Modified Adomian decomposition method for solving fractional optimal control problems," Transactions of the Institute of Measurement and Control, vol. 40, no. 6, pp. 2054-2061, 2018.

[21] B. B. İ. Eroğlu, D. Avc1, and N. Özdemir, "Constrained optimal control of a fractionally damped elastic beam," International Journal of Nonlinear Sciences and Numerical Simulation, vol. 21, pp. 389-395, 2020.

[22] F. Evirgen, "Analyze the optimal solutions of optimization problems by means of fractional gradient based system using VIM," An International Journal of Optimization and Control: Theories \& Applications (IJOCTA), vol. 6, no. 2, pp. 75-83, 2016.

[23] B. B. İ. Eroğlu, D. Avc1, and N. Özdemir, "Optimal control problem for a conformable fractional heat conduction equation," Acta Physica Polonica A, vol. 132, no. 3, pp. 658-662, 2017.

[24] L. Zhang and Z. Zhou, "Spectral Galerkin approximation of optimal control problem governed by Riesz fractional differential equation," Applied Numerical Mathematics, vol. 143, no. 6, pp. 247-262, 2019.

[25] A. B. Salati, M. Shamsi, and D. F. M. Torres, "Direct transcription methods based on fractional integral approximation formulas for solving nonlinear fractional optimal control problems," Communications in Nonlinear Science and $\mathrm{Nu}$ merical Simulation, vol. 67, pp. 334-350, 2019.

[26] S. J. Liao, "The proposed homotopy analysis technique for the solution of nonlinear problems," Ph. D. Thesis, Shanghai Jiao Tong University, Shanghai, China, 1992.

[27] D. Baleanu, B. Agheli, and R. Darzi, "An optimal method for approximating the delay differential equations of noninteger order," Advances in Difference Equations, vol. 1, p. 284, 2018.

[28] B. Pan, Y. Ma, and Y. Ni, "A new fractional homotopy method for solving nonlinear optimal control problems," Acta Astronautica, vol. 161, pp. 12-23, 2019.

[29] J. Biazar and R. Montazeri, "Optimal homotopy asymptotic and multistage optimal homotopy asymptotic methods for solving system of volterra integral equations of the second kind," Journal of Applied Mathematics, vol. 2019, Article ID 3037273, 17 pages, 2019.
[30] F. Jarad, E. Uğurlu, T. Abdeljawad, and D. Baleanu, "On a new class of fractional operators," Advances in Difference Equations, vol. 2017, p. 247, 2017.

[31] M. A. Zaky and J. A. T. Machado, "On the formulation and numerical simulation of distributed-order fractional optimal control problems," Communications in Nonlinear Science and Numerical Simulation, vol. 52, pp. 177-189, 2017.

[32] A. Hamdache, S. Saadi, and I. Elmouki, "Free terminal time optimal control problem for the treatment of HIV infection," An International Journal of Optimization and Control: Theories \& Applications (IJOCTA), vol. 6, no. 1, pp. 33-51, 2016.

[33] O. P. Agrawal, O. Defterli, and D. Baleanu, "Fractional optimal control problems with several state and control variables," Journal of Vibration and Control, vol. 16, no. 13, pp. 1967-1976, 2010. 